\title{
Gut eukaryotic communities in pigs: diversity, composition and host genetics contribution
}

\author{
Yuliaxis Ramayo-Caldas ${ }^{1 *}$ (D), Francesc Prenafeta-Boldú², Laura M. Zingaretti ${ }^{3}$, Olga Gonzalez-Rodriguez ${ }^{1}$,
} Antoni Dalmau', Raquel Quintanilla ${ }^{4}$ and Maria Ballester ${ }^{1}$

\begin{abstract}
Background: The pig gut microbiome harbors thousands of species of archaea, bacteria, viruses and eukaryotes such as protists and fungi. However, since the majority of published studies have been focused on prokaryotes, little is known about the diversity, host-genetic control, and contributions to host performance of the gut eukaryotic counterparts. Here we report the first study that aims at characterizing the diversity and composition of gut commensal eukaryotes in pigs, exploring their putative control by host genetics, and analyzing their association with piglets body weight.
\end{abstract}

Results: Fungi and protists from the faeces of 514 healthy Duroc pigs of two sexes and two different ages were characterized by $18 \mathrm{~S}$ and ITS ribosomal RNA gene sequencing. The pig gut mycobiota was dominated by yeasts, with a high prevalence and abundance of Kazachstania spp. Regarding protists, representatives of four genera (Blastocystis, Neobalantidium, Tetratrichomonas and Trichomitus) were predominant in more than the $80 \%$ of the pigs. Heritabilities for the diversity and abundance of gut eukaryotic communities were estimated with the subset of $60 \mathrm{~d}$ aged piglets $(N=390)$. The heritabilities of a-diversity and of the abundance of fungal and protists genera were low, ranging from 0.15 to 0.28 . A genome wide association study reported genetic variants related to the fungal a-diversity and to the abundance of Blastocystis spp. Annotated candidate genes were mainly associated with immunity, gut homeostasis and metabolic processes. Additionally, we explored the association of gut commensal eukaryotes with piglet body weight. Our results pointed to a positive contribution of fungi from the Kazachstania genus, while protists displayed both positive (Blastocystis and Entamoeba) and negative (Trichomitus) associations with piglet body weight.

Conclusions: Our results point towards a minor and taxa specific genetic control over the diversity and composition of the pig gut eukaryotic communities. Moreover, we provide evidences of the associations between piglets' body weight after weaning and members from the gut fungal and protist eukaryote community. Overall, this study highlights the relevance of considering, along with that of bacteria, the contribution of the gut eukaryote communities to better understand host-microbiome association and their role on pig performance, welfare and health.

Keywords: Gut eukaryotes, Fungi, Protists, Pig, Host genetics

\footnotetext{
* Correspondence: yuliaxis.ramayo@irta.cat

${ }^{1}$ Animal Breeding and Genetics Program, Institute of Agrifood Research and

Technology (IRTA), Torre Marimon, 08140 Caldes de Montbui, Spain

Full list of author information is available at the end of the article
}

(c) The Author(s). 2020 Open Access This article is licensed under a Creative Commons Attribution 4.0 International License, which permits use, sharing, adaptation, distribution and reproduction in any medium or format, as long as you give appropriate credit to the original author(s) and the source, provide a link to the Creative Commons licence, and indicate if changes were made. The images or other third party material in this article are included in the article's Creative Commons licence, unless indicated otherwise in a credit line to the material. If material is not included in the article's Creative Commons licence and your intended use is not permitted by statutory regulation or exceeds the permitted use, you will need to obtain permission directly from the copyright holder. To view a copy of this licence, visit http://creativecommons.org/licenses/by/4.0/. 


\section{Introduction}

The gut microbiome harbors thousands of species of archaea, bacteria, viruses and eukaryotes such as protists and fungi that contribute to host biology. The gut eukaryotic communities of monogastric species show a higher interindividual variability, but less abundance, diversity and richness than their bacterial counterparts [1]. The so called gut mycobiome of healthy humans is dominated by fungal genera like Saccharomyces, Candida, Penicillim, Aspergillus and Malassezia [2], while protist genera such as Blastocystis, Entamoeba and Enteromonas have been reported in the human gut across different geographical location [3-5]. There is currently considerable interest in understanding the mechanisms through which gut commensal eukaryotic communities contribute to host homeostasis and health [6]. The role of fungi and protists in the human gut remains poorly understood, but some authors suggest that commensal species may induce innate immune response in the host [7], and they could also have other potential benefits [5, 8, 9]. For example, Blastocystis spp. and non-pathogenic Entamoeba spp. have been associated with a healthy and highly diverse gut microbiome ecosystem $[6,10,11]$, while Tritrichomonas musculis modulates the intestinal immune system and increases host protection against mucosal bacterial infections in mice [12].

The few studies about gut eukaryotes conducted in pigs (Sus scrofa domesticus) used a limited sample size and mainly focused on isolated members of the eukaryote communities or parasite identification under pathogenic conditions [13-18]. In swine, Kazachstania spp. and members of the Saccharomycetaceae family are predominant in the gut mycobiota [13-16], while gut protist community of healthy pigs is dominated by Blastocystis spp., Tritrichomonas spp. and Balantidium coli. Environmental factors such as diet seems to play a key role in modulating the structure and composition of both eukaryote and prokaryote communities. However, little is known about the genetic control of the gut eukaryotes, since published studies have been focused on the role of host genetics in shaping the gut bacterial communities [19-21].

The main goal of this study is to characterize commensal fungi and protists inhabiting the gut of healthy pigs, explore the putative host genetic control over diversity and composition of gut eukaryotes communities, and analyze their association with piglets body weight.

\section{Results}

\section{Composition of the pig gut microbial eukaryotic communities}

The pig gut microbial eukaryote communities from 514 healthy pigs were analyzed by sequencing the $18 \mathrm{~S}$ rRNA gene and the ITS2 region. After quality control, 492 fungal and 227 protist ASVs were identified. The pig gut mycobiota was dominated by yeast from the Kazachstania genus, in particular by the species $K$. slooffiae and $K$. bovina (Fig. 1). Other yeasts associated to the Candida genus were identified, such as C. glabrata, C. albicans and $C$. (Diutinia) catenulata, which were present in $2-$ $7 \%$ of the animals (Additional file 1 Table S1). Besides, a moderate prevalence (15\% of pigs) of Tilletia puccinelliae was also observed (Additional file 1 Table S1). Along with the ascomycete Saccharomyces arboricola, a number of basidiomycetous yeasts were also detected with relatively low incidence $(<2 \%)$, such as Sporobolomyces roseus, Trichosporon dohaense, Debaryomyces prosopidis, Pichia sporocuriosa, Filobasidium globisporum and Vishniacozyma victoriae. Finally, the pig intestinal mycobiota included a third and more diverse group of cosmopolitan fungal species that are generally categorized as "soil fungi". In most cases they have a relatively low occurrence in gut $(<2 \%)$ and are primarily associated with a general saprotrophic ecophisiology (i.e. Aspergillus spp., Aureobasidium pullulans, Cladosporium tenuissimum, Mucor circinelloides and Penicillium polonicum).

With regard to protists, the prevalent ASV corresponded to an unidentified species of the subclass Trichostomatia (superphylum Alveolata in the SAR supergroup). Furthermore, the species Neobalantidium coli from this class was found in $82 \%$ of the animals (Additional file 1 Table S1). Likewise, with a relatively high incidence and average abundance (up to 99 and $29 \%$, respectively), a number of Blastocystis spp. (superphylum Stramenopiles in the SAR supergroup). Other detected commensal trichomonads included Tetratrichomonas buttreyi (94\% incidence), Hypotrichomonas imitans (67\% incidence), as well as the Tetratrichomonas strain PEKPR (10\% incidence), and with a very low incidence and abundance $(<1 \%)$, Tritrichomonas suis. Finally, one representative of the ameboid protist species Entamoeba gingivalis (supergroup Amoebozoa) was also detected with a relatively high incidence $(77 \%)$ but a low abundance $(<1 \%)$.

\section{Host and environmental factors modulating the diversity of pig gut microbial eukaryotes}

The modulatory effect of host and environmental factors over the diversity and composition of gut eukaryotic communities in pigs was evaluated with different approaches. Results from the first PERMANOVA with the whole dataset indicated that the combination of farm and animal age represented the most significant effect shaping the gut eukaryotic communities $(p<0.0001)$, explaining around 44 and $42 \%$ of the total variability of fungal and protist communities, respectively. The same data structure was recovered by the principal 


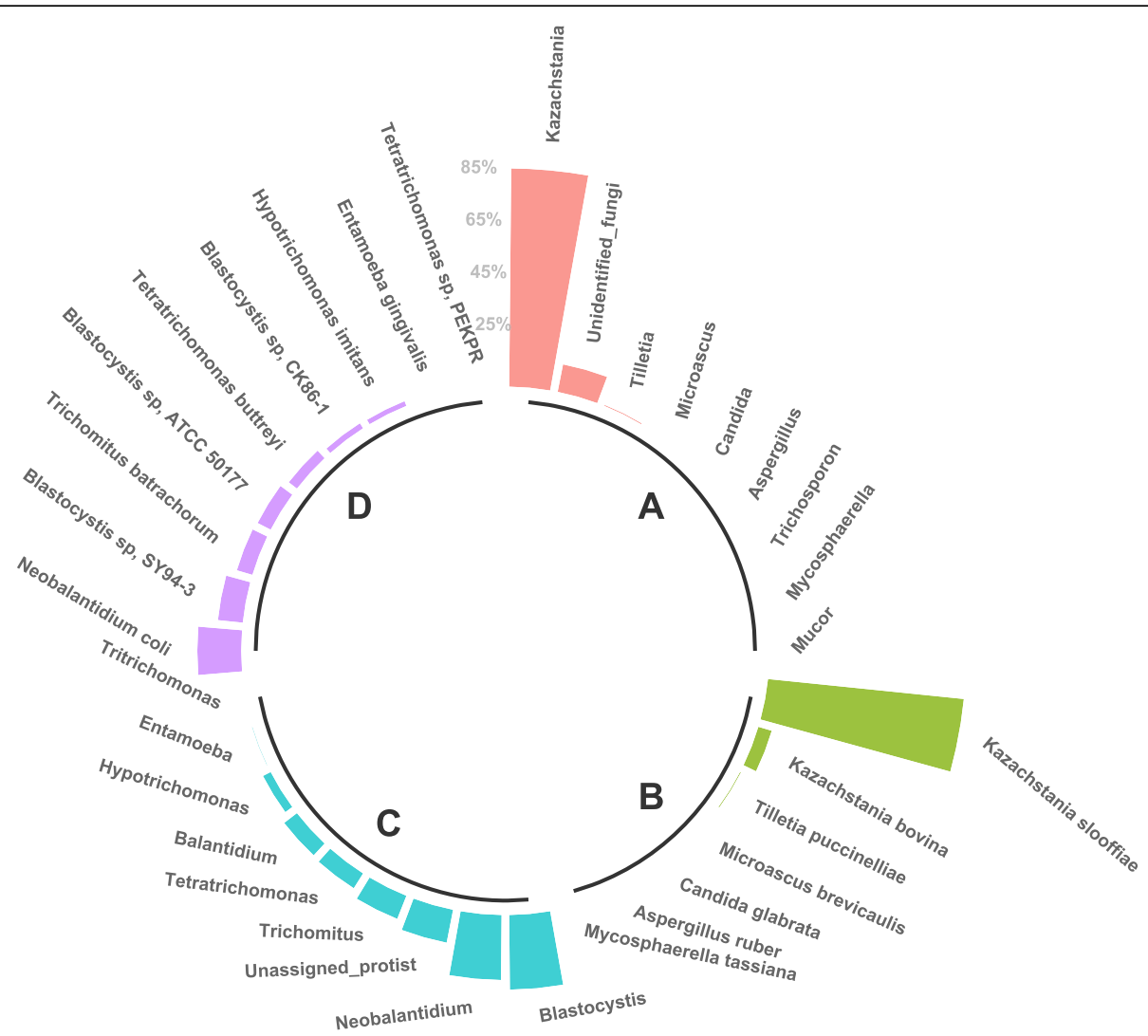

Fig. 1 Circular barplot representing the relative abundance of the most abundant fungal Genus (a) and species (b) as well as protists Genus (c) and species $(\mathbf{d})$

coordinates analysis (PCoA) based on the Bray-Curtis distance, which showed two clusters that match with sample farms origin (Additional file 2 Figure S1). The diversity index also revealed important differences between farms/ages (Kruskal-Wallis test). Samples taken at 190 days of age in the experimental farm showed a significantly higher protist $(p=0.013)$ and fungal $(p=$ 0.006) alpha-diversity (Additional file 2 Figure S1). In contrast, weaned piglets raised in commercial conditions showed higher protist beta-diversity. A second PERMANOVA within farm/age was performed. Results of weaned piglets in the commercial farm indicated an important effect of the batch ( $p$-value $<0.0001)$ on alphadiversity of both fungal and protist communities, whereas the pen effect in the second dataset (experimental farm) seems to affect exclusively protist diversity
(Table 1). Regarding sex effects, gut fungi alpha-diversity differed between sexes in weaned piglets ( $p$-value $=0.02$ ), whereas no differences between castrated males and females mycobiota diversity were observed at $190 \mathrm{~d}$ of age (Table 1).

Afterwards, we assessed the degree of host genetic control over the diversity and composition of gut protist and fungal communities by estimating their heritabilities. The estimated heritability of the $\alpha$-diversity index was low, with posterior mean estimates of ranging from 0.161 to 0.188 for fungi and protists, respectively (Table 2). The posterior highest density regions at 95\% encompassed values between 0.067 and 0.299 , indicating a probably very limited but not negligible heritability for these traits. In the same way, the heritabilities of most representative fungal and protist genera abundance reached low to moderate

Table 1 Resume of the permutational multivariate analysis of variance

\begin{tabular}{llll}
\hline Dataset & Community & Bacht R2 (P-value) & Sex R2 $(\boldsymbol{P}$-value $)$ \\
\hline Weaned piglets $(n=405)$ & Fungi & $0.12(p<0.001)$ & $0.01(p=0.02)$ \\
commercial farm & Protist & $0.07(p<0.001)$ & $0.02(p=0.60)$ \\
Finishing pigs $(n=109)$ & Fungi & $0.09(p=0.35)$ & $0.01(p=0.24)$ \\
experimental farm & Protist & $0.12(p=0.03)$ & $0.01(p=0.55)$ \\
\hline
\end{tabular}


Table 2 Mean (standard deviation) and highest density regions at 95\% of marginal posterior distributions for heritabilities of pig gut eukaryotes microbial traits

\begin{tabular}{cllll}
\hline Community & Level & Trait & Heritabiliy (SD) & HDR \\
\hline Fungi & a-diversity & Shannon-index & $0.161(0.053)$ & $0.067-0.266$ \\
& Genus & Kazachstania & $0.209(0.064)$ & $0.095-0.333$ \\
& Specie & Kazachstania_slooffiae & $0.216(0.071)$ & $0.093-0.353$ \\
Protist & Alpha-diversity & Shannon-index & $0.188(0.060)$ & $0.077-0.299$ \\
& Genus & Blastocystis & $0.281(0.101)$ & $0.096-0.483$ \\
& & Trichomitus & $0.233(0.077)$ & $0.085-0.383$ \\
& & Tetratrichomonas & $0.159(0.051)$ & $0.070-0.258$ \\
& Neobalantidium & $0.273(0.090)$ & $0.108-0.443$ \\
& Species & Trichomitus batrachorum & $0.240(0.075)$ & $0.100-0.382$ \\
& Tetratrichomonas buttrey & $0.158(0.048)$ & $0.070-0.256$ \\
& Neobalantidium coli & $0.256(0.086)$ & $0.100-0.425$ \\
& Blastocystis sp. ATCC 50177 & $0.245(0.086)$ & $0.086-0.409$ \\
& Blastocystis sp. CK86-1 & $0.203(0.066)$ & $0.081-0.325$ \\
\hline
\end{tabular}

(Table 2). The abundance of the protist Blastocystis genus was the most heritable (posterior mean 0.281, SD 0.101), followed by the abundance of Trichomitus (0.273, SD 0.090 ) and Neobalantidium (0.233, SD 0.077) genera. Also, a heritability around 0.209 was estimated for the abundance of the preponderant fungal genus Kazachstania, and lightly larger (0.216) for the abundance of Kazachstania slooffiae species.

\section{Identification of host genetic regions linked with the} composition and diversity of pig gut microbial eukaryotes Results from GWAS revealed few and weak association signals between the host genome and the gut microbial eukaryotes composition and diversity. We identified a total of 174 SNPs as significantly (at chromosome-wide level, FDR $<0.05)$ related with the abundance of protist community (Additional file 3 Table S2), located in seven intervals distributed across three pig Sus scrofa chromosomes: SSC6, SSC17 and SSC18 (Table 3). The $32.18 \%$ of these SNPs were intronic variants, $56.89 \%$ were intergenic, $5.75 \%$ were located upstream/downstream of genes, $1.15 \%$ were exonic synonymous, and 4\% mapped within non-coding transcript variants (Additional file 3 Table S2). Most of these associated SNPs (164 out of 174) were identified on SSC6 (Table 3 ), and were mainly associated with the relative abundance of two species of Blastocystis genera: CK86-1 and ATCC 50177 (Fig. 2). The remaining significant SNPs located on SSC17 (46.77-46.99 Mb interval) and SSC18 (two intervals: 4,53-4,57 $\mathrm{Mb}$ and $25,85-25,88 \mathrm{Mb}$ ) were also associated to the relative abundance of members of Blastocystis (Table 3). Regarding diversity, the aforementioned 141.91-145.39 $\mathrm{Mb}$ region of SSC6 resulted also associated with the fungi Shannon diversity-index (Fig. 2). Finally, no significant associations with either the relative abundance of fungi (Kazachstania genera or Kazachstania slooffiae) or the diversity of protist were observed.

\section{Genes and pathways associated to pig gut microbial eukaryotes}

A total of 229 protein-coding, 74 long non-coding RNA and two miRNAs (mir138-2 and ssc-mir-186) (Additional file 4 Table S3) were annotated within the host

Table 3 Resume of the chromosomal intervals identified by the GWAS

\begin{tabular}{lllll}
\hline Chr & Start $(\mathrm{bp})$ & End $(\mathrm{bp})$ & Size $(\mathrm{Mb})$ & Associated microbial trait \\
\hline 6 & $68,999,679$ & $69,466,937$ & 0.467 & Blastocystis sp. SY94-3 \\
6 & $120,437,893$ & $121,498,044$ & 1.060 & Blastocystis sp. ATCC 50177; Blastocystis sp. CK86-1 \\
6 & $122,778,463$ & $132,592,988$ & 9.814 & Blastocystis sp. ATCC 50177; Blastocystis sp. CK86-1 \\
6 & $141,914,558$ & $145,392,721$ & 3.478 & Blastocystis sp. ATCC 50177; Blastocystis sp. CK86-1; ITS a-diversity \\
17 & $46,764,111$ & $46,988,559$ & 0.224 & Blastocystis sp. \\
18 & $4,538,602$ & $4,570,818$ & 0.032 & Blastocystis sp. ATCC 50177 \\
18 & $25,850,358$ & $25,885,196$ & 0.035 & Blastocystis sp. ATCC 50177 \\
\hline
\end{tabular}



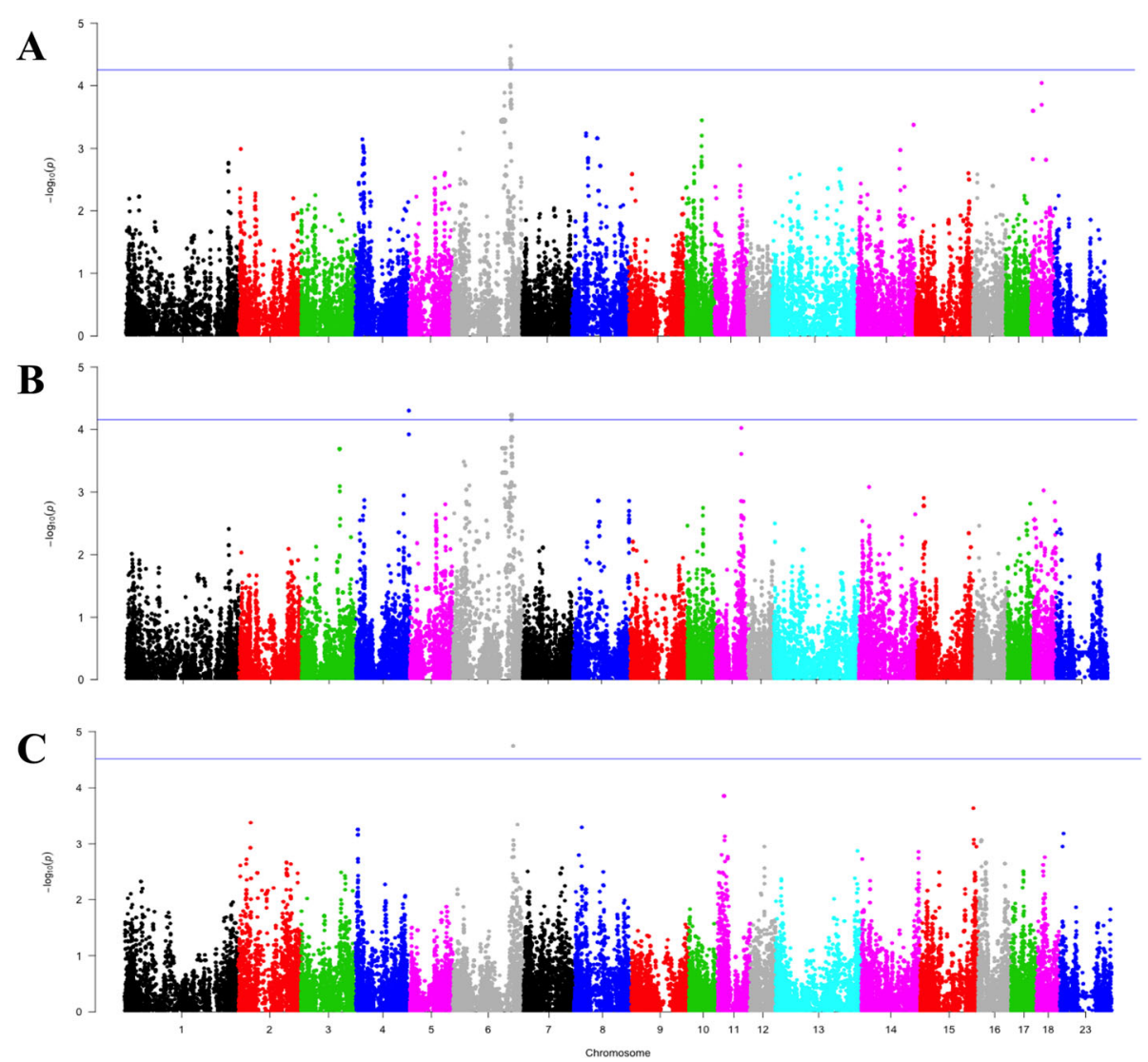

Fig. 2 Significance across the whole genome of the association between SNP markers and the relative abundance of (a) Blastocystis sp. ATCC 50177, (b) Blastocystis sp. CK8166 and (c) Fungi Alpha diversity. The x-axis represents the pig autosomal chromosomes (1-18), and the $y$-axis the significance level represented as the $-\log _{10}(P$-value)

genomic intervals associated to the gut microbial eukaryotes composition and diversity. A detailed exploration of annotated genes revealed that the Interleukin 23 Receptor gene (IL23R) holds two intronic SNPs, rs343576943 ( $p$-value $\left.=1,8^{*} 10^{-5}\right)$ and rs81392455 (pvalue $=1,8^{*} 10^{-5}$ ) that were associated with fungal $\alpha$ diversity. Furthermore, the variants rs81391297, rs81391299 and rs81299019, mapped within the 122.8$132.6 \mathrm{Mb}$ interval on SSC6 and associated with the relative abundance of Blastocystis spp., were located on intron eight of the Phosphatidylinositol 3-Kinase Catalytic Subunit Type 3 (PIK3C3) gene. On SSC17, the intronic variant rs331945396 (SSC17:46832698-46,832,698) in the Hepatocyte Nuclear Factor 4 Alpha (HNF4A) gene was also associated with the relative abundance of Blastocystis genera $\left(\mathrm{p}\right.$-value $\left.=9,02 * 10^{-5}\right)$. Other genes related to the immune system were annotated within the aforementioned chromosomal intervals, as for example the TNF Receptor Superfamily Member 9 (TNFRSF9), the Interleukin 12 Receptor Subunit Beta 2 (IL12RB2) or the Phosphatidylinositol-4,5-Bisphosphate 3-Kinase Catalytic Subunit Delta $(P I K 3 C D)$, as well as other members of immune-related pathways (e.g. CDH1, CDH11, CDH3,
CDH5, LPAR3, PIK3C3, CCL17, CCL22, CDH5, CKLF and $M M P 15)$. Finally, the functional annotation revealed that these genes belong to a variety of physiological processes and gene networks related to Cell-To-Cell Signaling and Interaction, Cellular Assembly and Organization, Cellular Function and Maintenance, Developmental Disorder, Hereditary Disorder, Cellmediated Immune Response, Humoral Immune Response, Immune Cell Trafficking, and Protein Synthesis (Additional file 5 Table S4). Metabolic pathways most significantly enriched by the list of candidate genes include Relaxin Signaling, Leptin Signaling in Obesity, IGF-1 Signaling, PXR/RXR Activation and HIF1 $\alpha$ Signaling pathways. It should be noted the overrepresentation of immune-related pathways such as Go12/13 Signaling, IL-9 Signaling, IL-23 Signaling, IL-12 Signaling, ERK/ MAPK Signaling, Agranulocyte and Granulocyte Adhesion and Diapedesis (Additional file 6 Table S5).

\section{Association between pig gut eukaryotes and host performance}

Most relevant fungal and protist genus associated with body weight are summarized in Fig. 3. The result 
suggested association between piglets' body weight and the abundance of Kazachstania fungal genera as well as protist members of Entamoeba, Trichomitus, Tetratrichomonas and Blastocystis. The Shapley Additive Explanation (SHAP) values showed a nonlinear association between Kazachstania and Blastocystis abundance and piglets body weight (Additional file 7 Figure S2). Furthermore, Entamoeba on one side, and Trichomitus on the other, were positive and negatively associated with body weight (Additional file 7 Figure S2).

\section{Discussion}

The analysis of gut microbial eukaryotic communities in 514 healthy animals have allowed characterizing commensal fungi and protists inhabiting the porcine gut tract. In agreement with previous reports in pigs [22-24] our results evidenced that pigs gut mycobiota is dominated by the yeast Kazachstania spp. which corresponds to the teleomorphic state of Candida slooffiae and Candida bovina [25]. Particularly the species K. slooffiae was found in all studied animals across farms, sex and ages, observation that is in agreement with the ubiquitous detection of this yeast from the gut of healthy pigs, and further supports the hypothesis that pigs intestinal tract is the primary ecological niche for $K$. slooffiae [13]. The closely related $K$. bovina was detected in the $45 \%$ of the studied pigs, and might also play a significant role in the gut. Several transient fungi that probably arise from the feeding or the environment were observed but with low incidence. These basidiomycetous yeasts have often been isolated from the phyllosphere [26] and are also known to cause human opportunistic infections in certain cases [27]. The plant-gut association for certain yeast is more evident in a second group of filamentous fungi that are characterized as specialized plant biotrophs. The most representative of those is the basidiomycete T. puccinelliae that, surprisingly, occurred in $15 \%$ of the studied pigs. Species from Tilletia genera are smut fungi that infect various grasses from the Poaceae family and encompass plant pathogens of economic importance in the

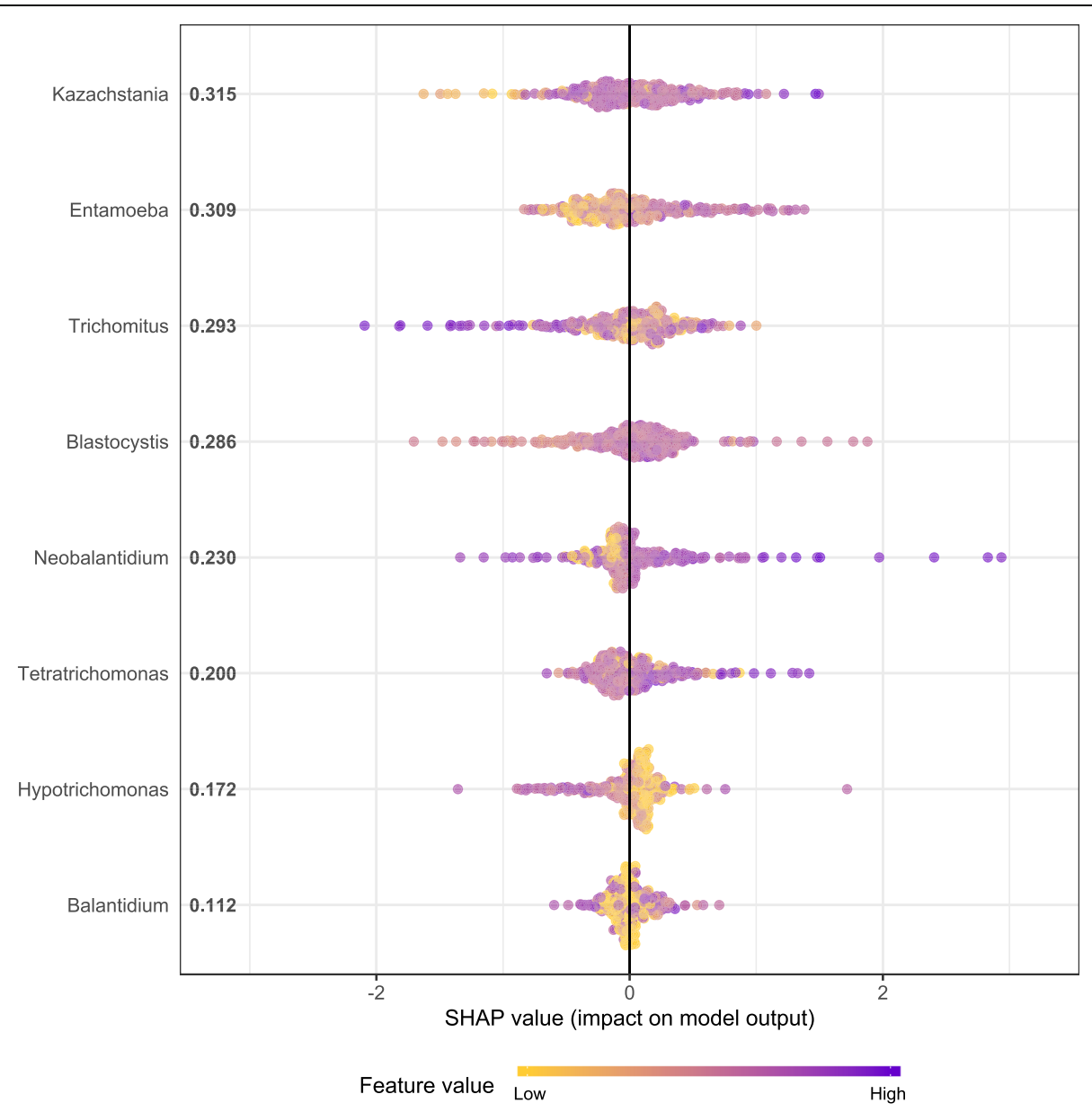

Fig. 3 Relevance of fungal and protist genera regarding piglets body weight, according to the Shapley Additive Explanation (SHAP) values. The Xaxis represents the SHAP value, the $y$-axis shows the genera names in order of importance from top to bottom, and the value correspond to the genera mean SHAP value 
production of cereals and forage grasses. So far, T. puccinelliae has only been isolated from weeping alkaligrass (Puccinellia distans), a common ruderal grass in Europe and North America [28]. Plants infected by other Tilletia species do not pose a toxicity risk for humans but contaminated grains might be derived to animal feed due to off-flavors [29]. Such association between feed and fungal gut might also apply for the plant pathogens Ustilago hordei and Mycosphaerella tassiana, found in about 3\% of the animals. Former results are in agreement with previous reports on humans and non-human primates $[2,30-32]$, and suggest that, differently to pig gut prokaryotes [33], the pig gut mycobiome may lack a stable core. Consequently, it is expected that a large proportion of the fungi detected in pig fecal samples may be transients from dietary or environmental origin. Meanwhile, the prevalence ( $>80 \%$ of the 514 pig samples) of four protist genera (Blastocystis, Neobalantidium, Tetratrichomonas and Trichomitus) was observed, which is in agreement with previous reports in pigs [17, 34]. Neobalantidium is a world-wide parasitic-opportunistic human pathogen that is transmitted through the fecal-oral route, particularly when there is a close contact with pigs, which are its asymptomatic reservoir hosts [35]. Blastocystis is a ubiquitous protozoan of human and pigs, commonly found in healthy populations $[6,36]$. However, several species in Blastocystis are causative agents of diarrhea in humans through the fecal-oral infection route, being the pig a common reservoir [37]. A number of protists in the Trichomonadida order (supergroup Excavata) that are common in the digestive tract of pigs [38] were also found in the present study. Colonization with this species has not been associated to virulence in mammals, but the full pathogenic potential of $T$. batrachorum has yet to be explored.

The analysis of host intrinsic and extrinsic factors ascertained the significant modulatory role of the environmental effects gathered in batch and farm factors (e.g. climate, management conditions, diet) on gut eukaryotes composition and diversity. Also important differences between the diversity of gut eukaryotic communities in weaned and finishing pigs were evidenced, whereas scarce differences due to the sex of the animal were observed. We explored the putative host genetic control of the diversity and composition of both gut protist and fungal communities. Despite the relatively low heritability estimates (posterior means from 0.158 to 0.281 ), they represent the first reported evidence of certain host genetic determinism of gut eukaryotic profile in pigs. The abundance of the protist Blastocystis genus was the most heritable, with around $28 \%$ of their variability explained by the genetic variability of the host, followed by the abundance of Trichomitus and Neobalantidium genus, and the preponderant fungal genus Kazachstania. Lower but not negligible heritabilities were obtained for a particularly complex trait such as the $\alpha$-diversity index in both protist and fungi, which allows hypothesizing that pigs can have a limited genetic tendency to harbor more or less diverse eukaryotic communities in its gut. These results should be taken with caution because of the limited sample size, but as a whole they allow inferring a limited and taxa specific genetic control over diversity and composition of gut eukaryotes. Besides, GWAS revealed genetic variants associated at chromosomal level with fungal $\alpha$-diversity (on SSC6) and the abundance of Blastocystis spp. (on SSC6, 17 and 18). Two intronic SNPs associated with fungal $\alpha$-diversity were mapped on the IL23R gene. Remarkably, this gene, which is highly expressed in Th17 cells, seems to play an important role in the proliferation and survival of these cells, which are critical for host defense against fungal infections [39]. In fact, genetic variants in the IL23R gene have been associated with different susceptibility to fungal infections [40] and $I L-23 R$ deficient mouse was susceptible to systemic infection with $C$. albicans [41]. Furthermore, IL-23R had been reported to be involved in the diversity of ileum microbiota in humans [42]. In the same chromosome, PIK3C3 gene gathered two SNPs associated to Blastocystis abundance. PIK3C3 is required for $\mathrm{T}$ cell homeostasis [43-45] and reported to play a relevant role in maintaining gut homeostasis [46]. Other genes involved in the intestinal epithelial integrity and gut homeostasis were annotated on SSC17, such as HNF4A, a relevant regulator that mediates microbial control of intestinal gene expression [47]. Also a number of genes related to the immune system were annotated within the aforementioned chromosomal intervals, as the TNFRSF9 gene that contributes to the development, survival and activation of T cells [48, 49], the IL12RB2 and PIK3CD genes, and a plethora of members of the immune-related pathways Go12/13 Signaling, IL-9 Signaling, IL-23 Signaling, IL-12 Signaling, ERK/MAPK Signaling, Agranulocyte and Granulocyte Adhesion and Diapedesis. Several studies, most of them performed in cell lines, have tried to shed light on Blastocystis-host interactions. These studies have shown that Blastocystis are able to disrupt intestinal barrier integrity and function, and that they modulate the host immune response through the degradation of IgA, the suppression of iNOS, and the induction of proinflammatory cytokines such as IL-12 and IL-23, among others [50, 51]. Furthermore, Blastocystis subtypedependent upregulation of pro-inflammatory cytokines in macrophages through the activation of MAPK pathways has been described [52]. Overall, it is noteworthy to highlight that in our study genes related to pathways previously associated with Blastocystis experimental infections were identified as being associated with Blastocystis relative abundance. Although further genetic and 
functional analyses are needed to better understand how the biological mechanisms of the host could modulate the diversity and abundance of fungi and protists, and their relationship with the gut bacterial counterparts. These findings are in line with recent reports in mice [53], and suggest that polymorphisms located in (or in linkage disequilibrium with) genes related to immune system and gut homeostasis may modulate the diversity and composition of the eukaryote gut communities in pigs. The evolutionary mechanism beyond the observed association may be explained by indirect relationships between mutual favorable selection at host-genome and microbial level $[54,55]$. Although far to be fully understood, some examples of host-genetic microbiota associations have been reported (reviewed in [55]), that similar to our results identify candidate genes mainly related to host-response against pathogens, sensing microbes, cell signaling pathways and innate immune system. However, in agreement with previous reports centered in gut bacterial $[53,56]$, our findings also indicate a limited and target specific host genetic control over the composition of the gut eukaryote communities in pig.

On another level, we provided evidences of the association between piglets body weight after weaning and members from the fungal and protists gut eukaryote community. A previous study with growing piglets, reported no significant effects on growth performance parameters upon oral supplementation with $K$. slooffiae [57]. However, a recent detailed metabolic study conducted by the same authors [58] under laboratory conditions with cultures of $K$. slooffiae, concluded that this yeast produces peptides and short chain fatty acids that might benefit the gut health, and provides an additional protein source that contains essential amino acids and other useful growth factors for the animal [58]. Consequently, it seems plausible that the observed relationships between Kazachstania and piglets body weight partially relies on the $K$. slooffiae positive effect on gut health through the production of essential amino acids and short fatty acids that are absorbed by the host or employed by others microbial members of the pig gut microbiome. On the other hand, the impact of members of the gut eukaryotic community to growth performance and the digestive enzyme activities have been reported in shrimp [59]. Furthermore, Blastocystis spp. and non-pathogenic Entamoeba spp. have been associated with a healthy and diverse gut microbiome ecosystem $[6$, 10, 11]. Likewise, mechanisms of horizontal gene transfer of genes associated with cellulose degradation and carbohydrate use from bacterial to protist such as Blastocystis have been documented [60-62]. Therefore, we hypothesize that observed positive relationships between piglets body weight at 60 days and members of Blastocystis and Entamoeba may be explained by the capacity of Blastocystis to degrade carbohydrates, which are routinely included after weaning in a typical transition diet. An alternative explanation may be also the recognized $[6,10$, 11,62] positive effects of Blastocystis and Entamoeba over the microbiota diversity and richness, which is turn is associated with host gut health.

In summary, our findings highlight the relevance of considering the gut eukaryotic communities to better understand the porcine gastrointestinal microbiome and its contributions to host performance and health. At that point we cannot neglect the contribution of other genera that might be relevant at different ages, under different environmental conditions of for other phenotypic traits. We are also aware of some limitations of our study, as for example the limited sample size or the lack of degrees of freedom to estimate all effects, but also of methodological constraints such as primers choice or the use of less curated (compared to bacterial) protists and fungal databases, that together with the reduced amount of public available reference genomes compromise the accuracy of the taxonomic classification. To the best of our knowledge, this study represents the largest effort to characterize the gut fungal and commensal protist communities in pigs, but further larger studies including experimental validations and alternative meta-sequencing approaches are needed to unveil the role of the hostassociated microbial communities in pigs production performance, welfare and health.

\section{Conclusions}

The diversity and composition of gut commensal eukaryotic communities in healthy pigs at two ages have been characterized. The porcine gut mycobiota is dominated by yeast, with a high prevalence of Kazachstania, and a common set of four protist genera (Blastocystis, Neobalantidium, Tetratrichomonas and Trichomitus) persisted through the majority of animals. Our results point towards a minor and taxa specific genetic control over the diversity and composition of the pig gut eukaryotic communities, but we describe associations with genes functionally related to the immune system and gut homeostasis that might have an effect in modulating the fungi $\alpha$-diversity and the abundance of Blastocystis ssp. We provide also evidences of the associations between piglets body weight after weaning and members from the gut fungal and protist eukaryote community. Overall, our findings highlight the relevance of considering, together with that of bacteria, the contribution of gut eukaryotic communities to better understand the hostmicrobiome association and its role on pig performance, welfare and health.

\section{Methods}

Sample collection, DNA extraction and sequencing

Fecal samples were collected at two ages from 514 healthy Duroc pigs belonging to the same commercial 
outbred line but allocated in two different farms. A total of 405 weaned piglets (204 males and 201 females) distributed in seven batches were sampled in a commercial farm at $60 \pm 8$ days of age and mean body weight around $18.58 \mathrm{~kg}$ (SD 3.13), after 4 weeks receiving the same transition-based diet (Additional file 8 Table S6). The remaining 109 pigs (50 castrated males and 59 females) were raised under intensive standard conditions at IRTA experimental farm (IRTA, Monells, Spain), and fecal samples were collected at $190 \pm 5$ days of age, when they fed a finishing standard diet. Both groups of pigs were genetically connected. Animal care and experimental procedures were carried out following national and institutional guidelines for the Good Experimental Practices and were approved by the IRTA Ethical Committee.

DNA was extracted with the DNeasy PowerSoil Kit (QIAGEN, Hilden, Germany), following manufacturer's instructions. Extracted DNA was sent to the University of Illinois Keck Center for Fluidigm sample preparation and sequencing. The Internal Transcribed Spacer 2 (ITS2) region was amplified using primers ITS3: 5'GCATCGATGAAGAACGCAGC-3' and ITS4: 5'TCCTCCGCTTATTGATATGC-3' [63]. Protist-specific primers [64], F-566: 5' -CAGCAGCCGCGGTAATTCC3' and R-1200: 5' -CCCGTGTTGAGTCAAATTAAGC$3^{\prime}$ were used to amplify the $18 \mathrm{~S}$ rRNA gene fragment. Amplicons were paired-end $(2 \times 250 \mathrm{nt})$ sequenced on an Illumina NovaSeq (Illumina, San Diego, CA, USA).

\section{Bioinformatics and statistical analysis}

Sequences were analysed with Qiime2 [65], barcode sequences, primers and low-quality reads (Phred scores of $<30)$ were removed. The quality control also trimmed sequences based on expected amplicon length and removed chimeras. Afterwards, sequences were processed into Amplicon Sequences Variants (ASVs) at 99\% of identity. ASVs present in less than three samples and representing less than $0.005 \%$ of the total counts were filtered out. Samples with less than 6000 (fungi, $n=21$ samples) or 10,000 (protists) reads were also excluded. ASVs were classified to the lowest possible taxonomic level based on SILVA v123 database [66] for 18S rRNA genes, and the UNITE QIIME version (release 18.11.2018) for fungi [67]. Subsequently, we excluded those ASVs not taxonomically classified as protists or fungi. Moreover, we verified the fungi taxonomic assignation following the recommendation of Nilsson [68] by a manual examination of the most abundant fungal ASVs against the International Nucleotide Sequence Database (http://www.insdc.org/). Before the estimation of diversity indexes, samples were rarefied at 6000 (fungi) and 10,000 (protists) reads of depth, to allow an equal depth. Diversity metrics were estimated with vegan $\mathrm{R}$ package [69]. Alpha-diversity was evaluated with the
Shannon index [70], and Beta-diversity was assessed using the Whittaker index [71]. To identify environmental (farm, batch or pen) and host-covariates (sex, age, body weight) that may modulate the diversity, structure and profile of the eukaryote communities, we run a Permutational multivariate analysis of variance (PERMANOVA) test using the adonis function from vegan [69]. Significance levels were determined after 10,000 permutations and the multiple comparison tests were performed using False Discovery Rate (FDR).

\section{Genotype data and estimation of heritability}

The putative host genetics determinism of gut eukaryotic profile in pigs was initially assessed by estimating the heritability $\left(h^{2}\right)$ of both fungi and protists alpha-diversity, as well as of their taxa abundance. For these analyses, we used the samples taken at $60 \pm 8$ days of age in the commercial farm. The Porcine $70 \mathrm{k}$ GGP Porcine HD Array (Illumina, San Diego, CA) was used to genotype 390 out of 405 animals sampled in the commercial farm. Parameters estimation was performed using the following Bayesian mixed model implemented with the bglr R package [72]:

$$
y_{i j k}=s e x_{j}+b_{k}+u_{i}+e_{i j k}
$$

where $y_{i j k}$ corresponds to the alpha-diversity (fungi or protist) or taxa (genera or specie) clr-transformed abundance of the th individual of sex $j$ in the $k^{\text {th }}$ batch; sex $x_{j}$ and $b_{k}$ correspond to the systematic effects of $j^{\text {th }}$ sex $(2$ levels) and $k^{\text {th }}$ batch effect (7 levels), respectively; $u_{\mathrm{i}}$ is the random genetic effect of individual $i$, distributed as case $\sim(0,2)$ being the genomic relationship matrix calculated using the filtered autosomal SNPs based on the methodology of Yang et al. [73]; finally, $e_{i j k}$ is the random residual term, with a distribution $\mathbf{e} \sim(0, \mathbf{I}$ $\left.\sigma_{e}^{2}\right)$. The model was run using a Gibbs sampler with 30,000 iterations and a burn-in of 2000 rounds. Posterior sample mean and standard deviation of the heritability ( $\left.h^{2}=\sigma_{u}^{2} /\left(\sigma_{u}^{2}+\sigma_{e}^{2}\right)\right)$ were obtained from the resulted posterior distributions.

\section{Genome wide association study (GWAS)}

Quality control was performed with plink [74] to exclude single nucleotide polymorphisms (SNPs) with minor allele frequencies $<5 \%$, rates of missing genotypes above $10 \%$, as well as SNPs that did not map to the porcine reference genome (Sscrofa11.1 assembly). Then, to identify SNPs from the host genome associated with the alpha-diversity as well as protists and fungi relative abundances, genome-wide association studies (GWAS) were performed between 42,608 SNPs and the alphadiversity or the centered log ratio (clr) transformed genera and species abundance tables. Only the genera and 
species fully taxonomically classified and present in more than $80 \%$ of the samples were included in the analysis. For this propose, the genome-wide complex trait analysis (GCTA) software [73] was employed using the following model at each SNP:

$$
y_{i j k}=s e x_{j}+b_{k}+u_{i}+s_{l i} a_{l}+e_{i j k}
$$

where $y_{i j k}$ corresponds to the alpha-diversity (fungi or protist) or taxa (genera or specie) clr-transformed abundance of the ${ }^{\text {th }}$ individual of sex $j$ in the $\mathrm{k}^{\text {th }}$ batch; $\operatorname{sex}_{j}$, $b_{k}$ and $u_{i}$ are, respectively, the effects of sex, batch and infinitesimal genetic effect described in the previous model; $s_{l i}$ is the genotype (coded as $\left.0,1,2\right)$ for the $l^{\text {th }}$ SNP of individual $i$, and $a_{l}$ is the allele substitution effect of SNP $l$ on the analysed trait. A SNP was considered to be significantly associated if the corresponding $p$-value was lower than 0.05, after Benjamini-Hochberg [75] correction for multiple testing at chromosome level.

\section{Gene functional classification and canonical pathway analyses}

Functional classification and pathway analyses of the annotated candidate genes were carried out using the Ingenuity Pathways Analysis software (IPA; Ingenuity Systems, http://www.ingenuity.com). Significance levels for enrichment of each canonical pathway in the list of candidate genes were calculated using Fisher's exact test, and the resulting $p$-values were corrected for multipletest using the Benjamini and Hochberg algorithm [75]. The cut-off for considering an enrichment as significant was established at a corrected $\mathrm{p}$-value $<0.05$.

\section{Association between pig gut eukaryote communities and body weight}

The associations between fungal and protist genera abundance and piglest body weight at 60-days was evaluated using the dataset from the commercial farm $(n=$ 405). In a first step we implemented XGBoost, a fast algorithm which incorporates a penalization term in the loss function to prevent overfitting (Chen \& Guestrin, 2016) using the XGBoost R packages. Afterwards, the importance of the variables was evaluated with Shapley Additive Explanation (SHAP) algorithm. SHAP (Lundberg \& Lee, 2017) is an extension of the coalition game from the game theory (Shapley, 1953), which is used in a context of multicollinearity. Basically, the model evaluates the importance of each variable by measuring a conditional contribution, i.e., the effect caused in the prediction by including that variable. SHAP values were obtained and visualized using the ShapXGboost R package [76].

\section{Supplementary information}

Supplementary information accompanies this paper at https://doi.org/10. 1186/s42523-020-00038-4

Additional file $\mathbf{1}$ Table S1. Description of the prevalence and relative abundance of the pig gut eukaryotes communities.

Additional file 2 Figure $\mathbf{S 1}$. Sample distribution of fungi (A) and protist (B) communities. Diversity indexes of fungi (C) and protist (D) communities. Blue color represents the finishing pigs (experimental farm at 190 days) and red the weaned piglets (commercial farm at 60 days).

Additional file $\mathbf{3}$ Table S2. Description of the genetic markers identified as significantly according to the genome-wide association studies.

Additional file $\mathbf{4}$ Table S3. Description of the genes annotated within the genomic intervals.

Additional file $\mathbf{5}$ Table S4. Functional annotation of the identified candidate genes.

Additional file 6 Table S5. Metabolic pathways significantly enriched by the list of candidate genes.

Additional file 7 Figure S2. Patterns of association between the piglets body weight at 60-days old and the fungal and protist abundance according to the Shapley Additive Explanation (SHAP) values. The x-axis represent the genera abundance after Cumulative Sum Scaling (CSS) normalization and log transformation. Y-axis represents the SHAP values of the piglets' body weight.

Additional file $\mathbf{8}$ Table S6. Description of the ingredients and nutrient content of the diets used during the experiment.

\section{Abbreviations}

QIIME: Quantitative insights into microbial ecology; ITS: Internal transcribed spacer; ASV: Amplicon sequence variant; clr: Centered log ratio

transformation; GWAS: Genome-wide association studies; XGBoost: eXtreme Gradient Boosting; FDR: False discovery rate

\section{Acknowledgements}

The authors warmly thank all technical staff from Selección Batallé S.A, for providing the animal material and their collaboration during the sampling, as well as to Juan Pablo Sanchez for valuable discussions and comments on the manuscript.

\section{Authors' contributions}

$Y R C, M B, A D$, and $R Q$ designed the study. $Y R C, M B, O G$ and $R Q$ performed the sampling. $M B$ and $O G$ carried out DNA extraction. YRC, FPB and $L Z$ analyzed the data. $Y R C, F P B, L Z, M B$ and $R Q$ interpreted the results and wrote the manuscript. The authors read and approved the final version of the manuscript.

\section{Funding}

YRC was funded by Marie Skłodowska-Curie grant (P-Sphere) agreement No 6655919 (EU). LMZ is recipient of a Ph.D. grant from Ministry of Economy and Science, Spain associated with 'Centro de Excelencia Severo Ochoa 2016-2019' award SEV-2015-0533 to CRAG. MB is recipient of a Ramon y Cajal post-doctoral fellowship (RYC-2013-12573) from the Spanish Ministry of Economy and Competitiveness. Part of the research presented in this publication was funded by Grants AGL2016-75432-R, AGL2017-88849-R awarded by the Spanish Ministry of Economy and Competitiveness. The authors belong to Consolidated Research Group TERRA (AGAUR, ref. 2017 SGR 1290 and 2017 SGR 1719).

\section{Availability of data and materials}

The raw sequencing data employed in this article has been submitted to the NCBI's sequence read archive (https://www.ncbi.nlm.nih.gov/sra); BioProject: PRJNA608629.

\section{Ethics approval}

Animal care and experimental procedures were carried out following national and institutional guidelines for the Good Experimental Practices and were approved by the IRTA Ethical Committee. 


\section{Consent for publication}

Not applicable.

\section{Competing interests}

The authors declare no competing interests.

\section{Author details}

${ }^{1}$ Animal Breeding and Genetics Program, Institute of Agrifood Research and Technology (IRTA), Torre Marimon, 08140 Caldes de Montbui, Spain. ${ }^{2} \mathrm{GIRO}$, IRTA, Torre Marimon, 08140 Caldes de Montbui, Spain. ${ }^{3}$ Centre for Research in Agricultural Genomics, CSIC-IRTA-UAB-UB Consortium, Bellaterra, Spain. ${ }^{4}$ Animal Welfare Subprogram, IRTA, 17121 Monells, Spain.

Received: 3 March 2020 Accepted: 29 April 2020

\section{Published online: 07 May 2020}

\section{References}

1. Hooks KB, O'Malley MA. Contrasting strategies: human eukaryotic versus bacterial microbiome research. J Eukaryot Microbiol. 2019;67(2):279-95.

2. Nash AK, Auchtung TA, Wong MC, Smith DP, Gesell JR, Ross MC, et al. The gut mycobiome of the human microbiome project healthy cohort. Microbiome. 2017;5(1):153.

3. Parfrey LW, Walters WA, Lauber CL, Clemente JC, Berg-Lyons D, Teiling C, et al. Communities of microbial eukaryotes in the mammalian gut within the context of environmental eukaryotic diversity. Front Microbiol. 2014;5: 298.

4. Hamad I, Raoult D, Bittar F. Repertory of eukaryotes (eukaryome) in the human gastrointestinal tract: taxonomy and detection methods. Parasite Immunol. 2016:38(1):12-36.

5. Laforest-Lapointe I, Arrieta M-C. Microbial eukaryotes: a missing Link in gut microbiome studies. mSystems. 2018;3(2):e00201-17.

6. Chabé M, Lokmer A, Ségurel L. Gut protozoa: friends or foes of the human gut microbiota? Trends Parasitol. 2017;33(12):925-34.

7. Underhill DM, lliev ID. The mycobiota: interactions between commensal fungi and the host immune system. Nat Rev Immunol. 2014;14(6):405-16.

8. Parfrey LW, Walters WA, Knight R. Microbial eukaryotes in the human microbiome: ecology, evolution, and future directions. Front Microbiol. 2011;2:153.

9. Lukeš J, Stensvold CR, Jirků-Pomajbíková K, Wegener Parfrey L. Are human intestinal eukaryotes beneficial or commensals? PLoS Pathog. 2015;11(8): e1005039.

10. Audebert C, Even G, Cian A, Safadi DE, Certad G, Delhaes L, et al. Colonization with the enteric protozoa Blastocystis is associated with increased diversity of human gut bacterial microbiota. Sci Rep. 2016;6(1): 25255.

11. Tito RY, Chaffron S, Caenepeel C, Lima-Mendez G, Wang J, Vieira-Silva S, et al. Population-level analysis of Blastocystis subtype prevalence and variation in the human gut microbiota. Gut. 2019;68(7):1180-9.

12. Chudnovskiy A, Mortha A, Kana V, Kennard A, Ramirez JD, Rahman A, et al Host-protozoan interactions protect from mucosal infections through activation of the Inflammasome. Cell. 2016;167(2):444-456.e414.

13. Summers KL, Frey JF, Ramsay TG, Arfken AM. The piglet mycobiome during the weaning transition: a pilot study1. J Anim Sci. 2019;97(7):2889-900.

14. White JK, Nielsen JL, Madsen AM. Microbial species and biodiversity in settling dust within and between pig farms. Environ Res. 2019;171:558-67.

15. Arfken AM, Frey JF, Ramsay TG, Summers KL. Yeasts of burden: exploring the Mycobiome-Bacteriome of the piglet Gl tract. Front Microbiol. 2019;10: 2286.

16. Shieban F. Studies on intestinal protozoa of domestic pigs in the Teheran area of Iran**this project is supported in part by funds for the endemic diseases research project of plan organization and of the Department of Health Science, Teheran University School of Medicine. Br Vet J. 1971;127(3): iii-v.

17. Wylezich C, Belka A, Hanke D, Beer M, Blome S, Höper D. Metagenomics for broad and improved parasite detection: a proof-of-concept study using swine faecal samples. Int J Parasitol. 2019;49(10):769-77.

18. Matsubayashi M, Suzuta F, Terayama Y, Shimojo K, Yui T, Haritani M, et al. Ultrastructural characteristics and molecular identification of Entamoeba suis isolated from pigs with hemorrhagic colitis: implications for pathogenicity. Parasitol Res. 2014;113(8):3023-8.
19. Chen C, Huang X, Fang S, Yang H, He M, Zhao Y, et al. Contribution of host genetics to the variation of microbial composition of cecum lumen and feces in pigs. Front Microbiol. 2018;9:2626.

20. Camarinha-Silva A, Maushammer M, Wellmann R, Vital M, Preuss S, Bennewitz J. Host genome influence on gut microbial composition and microbial prediction of complex traits in pigs. Genetics. 2017;206(3):1637.

21. Crespo-Piazuelo D, Migura-Garcia L, Estellé J, Criado-Mesas L, Revilla M, Castelló A, et al. Association between the pig genome and its gut microbiota composition. Sci Rep. 2019;9(1):8791.

22. Hu J, Nie Y, Chen J, Zhang Y, Wang Z, Fan Q, et al. Gradual changes of gut microbiota in weaned miniature piglets. Front Microbiol. 2016;7:1727.

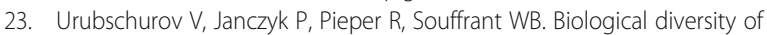
yeasts in the gastrointestinal tract of weaned piglets kept under different farm conditions. FEMS Yeast Res. 2008;8(8):1349-56.

24. Van Uden N, do Carmo Sousa L. Quantitative aspects of the intestinal yeast Flora of swine. Microbiology. 1962;27(1):35-40

25. Kurtzman CP, Robnett CJ, Ward JM, Brayton C, Gorelick P, Walsh TJ. Multigene phylogenetic analysis of pathogenic candida species in the Kazachstania (Arxiozyma) telluris complex and description of their ascosporic states as Kazachstania bovina sp. nov., K. heterogenica sp. nov., K. pintolopesii sp. nov., and K. slooffiae sp. nov. J Clin Microbiol. 2005;43(1): 101-11.

26. Kemler M, Witfeld F, Begerow D, Yurkov A. Phylloplane yeasts in temperate climates. In: Buzzini P, Lachance M-A, Yurkov A, editors. Yeasts in natural ecosystems: diversity. Cham: Springer International Publishing; 2017. p. 171-

27. Hoog GSD, Guarro J, Gene J, Figueras MJ. Atlas of clinical fungi. 2nd ed; 2000

28. Bao X, Carris LM, Huang G, Luo J, Liu Y, Castlebury LA. Tilletia puccinelliae, a new species of reticulate-spored bunt fungus infecting Puccinellia distans. Mycologia. 2010;102(3):613-23.

29. Preugschat K, Kersten S, Ettle T, Richter W, Karl H, Breves G, et al. Effects of feeding diets containing increasing proportions of bunt-infected wheat (Tilletia caries) on performance and health of pigs. Arch Anim Nutr. 2014; 68(1):55-62

30. Raimondi S, Amaretti A, Gozzoli C, Simone M, Righini L, Candeliere F, et al. Longitudinal survey of fungi in the human gut: ITS profiling, phenotyping, and colonization. Front Microbiol. 2019;10:1575.

31. Hallen-Adams HE, Kachman SD, Kim J, Legge RM, Martínez I. Fungi inhabiting the healthy human gastrointestinal tract: a diverse and dynamic community. Fungal Ecol. 2015;15:9-17.

32. Mann AE, Mazel F, Lemay MA, Morien E, Billy V, Kowalewski M, et al. Biodiversity of protists and nematodes in the wild nonhuman primate gut. ISME J. 2019;14:609-22.

33. Xiao L, Estellé J, Kiilerich P, Ramayo-Caldas Y, Xia Z, Feng Q, et al. A reference gene catalogue of the pig gut microbiome. Nat Microbiol. 2016; 1(12):16161.

34. Pakandl M. The prevalence of intestinal protozoa in wild and domestic pigs Vet Med (Praha). 1994;39(7):377-80.

35. Schuster FL, Ramirez-Avila L. Current world status of \&lt;em\&gt;Balantidium coli\&lt;/em\&gt. Clin Microbiol Rev. 2008:21(4):626.

36. Wang W, Bielefeldt-Ohmann H, Traub RJ, Cuttell L, Owen H. Location and pathogenic potential of Blastocystis in the porcine intestine. PLoS One. 2014;9(8):e103962

37. Rivera WL. Phylogenetic analysis of Blastocystis isolates from animal and human hosts in the Philippines. Vet Parasitol. 2008;156(3):178-82.

38. Mostegl MM, Richter B, Nedorost N, Lang C, Maderner A, Dinhopl N, et al. First evidence of previously undescribed trichomonad species in the intestine of pigs? Vet Parasitol. 2012;185(2-4):86-90.

39. Khader SA, Gaffen SL, Kolls JK. Th17 cells at the crossroads of innate and adaptive immunity against infectious diseases at the mucosa. Mucosal Immunol. 2009;2(5):403-11.

40. Carvalho A, Cunha C, Di lanni M, Pitzurra L, Aloisi T, Falzetti F, et al. Prognostic significance of genetic variants in the IL-23/Th17 pathway for the outcome of T cell-depleted allogeneic stem cell transplantation. Bone Marrow Transplant. 2010:45(11):1645-52

41. Nur S, Sparber F, Lemberg C, Guiducci E, Schweizer TA, Zwicky P, et al. IL-23 supports host defense against systemic Candida albicans infection by ensuring myeloid cell survival. PLoS Pathog. 2020;15(12):e1008115.

42. Zakrzewski M, Simms LA, Brown A, Appleyard M, Irwin J, Waddell N, et al. IL23R-protective coding variant promotes beneficial bacteria and diversity in 
the lleal microbiome in healthy individuals without inflammatory bowel disease. J Crohns Colitis. 2018;13(4):451-61.

43. Willinger T, Flavell RA. Canonical autophagy dependent on the class III phosphoinositide-3 kinase Vps34 is required for naive T-cell homeostasis. Proc Natl Acad Sci U S A. 2012;109(22):8670-5.

44. Parekh W, Wu L, Boyd KL, Williams JA, Gaddy JA, Olivares-Villagómez D, et al. Impaired autophagy, defective T cell homeostasis, and a wasting syndrome in mice with a T cell-specific deletion of Vps34. J Immunol. 2013; 190(10):5086-101.

45. McLeod IX, Zhou X, Li Q-J, Wang F, He Y-W. The class III kinase Vps34 promotes T lymphocyte survival through regulating IL-7Ra surface expression. J Immunol. 2011;187(10):5051.

46. Zhao S, Xia J, Wu X, Zhang L, Wang P, Wang $H$, et al. Deficiency in class III PI3-kinase confers postnatal lethality with IBD-like features in zebrafish. Nat Commun. 2018;9(1):2639.

47. Davison JM, Lickwar CR, Song L, Breton G, Crawford GE, Rawls JF. Microbiota regulate intestinal epithelial gene expression by suppressing the transcription factor hepatocyte nuclear factor 4 alpha. Genome Res. 2017; 27(7):1195-206.

48. Ward-Kavanagh LK, Lin WW, Šedý JR, Ware CF. The TNF receptor superfamily in co-stimulating and co-inhibitory responses. Immunity. 2016; 44(5):1005-19.

49. Schwarz H, Tuckwell J, Lotz M. A receptor induced by lymphocyte activation (ILA): a new member of the human nerve-growth-factor/tumor-necrosisfactor receptor family. Gene. 1993;134(2):295-8.

50. H. M, K.S T, H. Y. Blastocystis: pathogen or passenger? An evaluation of 101 years of research, vol. 4; 2012.

51. Ajjampur SSR, Tan KSW. Pathogenic mechanisms in Blastocystis spp. interpreting results from in vitro and in vivo studies. Parasitol Int. 2016;65(6, Part B):772-9.

52. Lim MX, Png CW, Tay CYB, Teo JDW, Jiao H, Lehming N, et al. Differential regulation of proinflammatory cytokine expression by mitogen-activated protein kinases in macrophages in response to intestinal parasite infection. Infect Immun. 2014;82(11):4789-801.

53. Khan AA, Yurkovetskiy L, O'Grady K, Pickard JM, de Pooter R, Antonopoulos DA, et al. Polymorphic immune mechanisms regulate commensal repertoire. Cell Rep. 2019;29(3):541-550.e544.

54. Zilber-Rosenberg I, Rosenberg E. Role of microorganisms in the evolution of animals and plants: the hologenome theory of evolution. FEMS Microbiol Rev. 2008;32(5):723-35.

55. Wang J, Chen L, Zhao N, Xu X, Xu Y, Zhu B. Of genes and microbes: solving the intricacies in host genomes. Protein Cell. 2018;9(5):446-61.

56. Rothschild D, Weissbrod O, Barkan E, Kurilshikov A, Korem T, Zeevi D, et al. Environment dominates over host genetics in shaping human gut microbiota. Nature. 2018:555(7695):210-5.

57. Urubschurov V, Büsing K, Freyer G, Herlemann DPR, Souffrant W-B, Zeyner A. New insights into the role of the porcine intestinal yeast, Kazachstania slooffiae, in intestinal environment of weaned piglets. FEMS Microbiol Ecol. 2016;93(2)..

58. Urubschurov V, Büsing K, Souffrant WB, Schauer N, Zeyner A. Porcine intestinal yeast species, Kazachstania slooffiae, a new potential protein source with favourable amino acid composition for animals. J Anim Physiol Anim Nutr. 2018;102(2):e892-901.

59. Dai W, Yu W, Zhang J, Zhu J, Tao Z, Xiong J. The gut eukaryotic microbiota influences the growth performance among cohabitating shrimp. Appl Microbiol Biotechnol. 2017;101(16):6447-57.

60. del Campo J, Bass D, Keeling PJ. The eukaryome: diversity and role of microeukaryotic organisms associated with animal hosts. Funct Ecol. 2019. Early view, Online Version of Record before inclusion in an issue.

61. Todaka N, Inoue T, Saita K, Ohkuma M, Nalepa CA, Lenz M, et al, Phylogenetic analysis of cellulolytic enzyme genes from representative lineages of termites and a related cockroach. PLoS One. 2010;5(1):e8636.

62. Eme L, Gentekaki E, Curtis B, Archibald JM, Roger AJ. Lateral Gene transfer in the adaptation of the anaerobic parasite Blastocystis to the gut. Curr Biol. 2017;27(6):807-20.

63. White TJ, Bruns T, Lee S, Taylor J. PCR protocols amplification and direct sequencing of fungal ribosomal RNA genes for phylogenetics: Elsevier; 1990.

64. Hadziavdic K, Lekang K, Lanzen A, Jonassen I, Thompson EM, Troedsson C. Characterization of the $18 \mathrm{~S}$ rRNA Gene for designing universal eukaryote specific primers. PLoS One. 2014;9(2):e87624.
65. Bolyen E, Rideout JR, Dillon MR, Bokulich NA, Abnet CC, Al-Ghalith GA, et al. Reproducible, interactive, scalable and extensible microbiome data science using QIIME 2. Nat Biotechnol. 2019;37(8):852-7.

66. Quast C, Pruesse E, Yilmaz P, Gerken J, Schweer T, Yarza P, et al. The SILVA ribosomal RNA gene database project: improved data processing and webbased tools. Nucleic Acids Res. 2013;41(Database issue):D590-6.

67. Community U. UNITE QIIME release for fungi. Version 18.11.2018. UNITE community; 2019. https://doi.org/10.15156/BIO/786334.

68. Nilsson RH, Anslan S, Bahram M, Wurzbacher C, Baldrian P, Tedersoo L. Mycobiome diversity: high-throughput sequencing and identification of fungi. Nat Rev Microbiol. 2019;17(2):95-109.

69. Jari Oksanen F, Blanchet G, Friendly M, Kindt R, Legendre P, McGlinn D, et al. Vegan: community ecology package. R package version 2.5-3. 2018. https://www.CRANR-projectorg/package=vegan.

70. Shannon C. A mathematical theory of communication; 1984

71. Whittaker RH. Evolution and measurement of species diversity. Taxon. 1972; 21(2/3):213-51.

72. Pérez P, de los Campos G. Genome-wide regression and prediction with the BGLR statistical package. Genetics. 2014;198(2):483-95.

73. Yang J, Lee SH, Goddard ME, Visscher PM. GCTA: a tool for genome-wide complex trait analysis. Am J Hum Genet. 2011;88(1):76-82.

74. Purcell S, Neale B, Todd-Brown K, Thomas L, Ferreira MAR, Bender D, et al. PLINK: a tool set for whole-genome association and population-based linkage analyses. Am J Hum Genet. 2007;81(3):559-75.

75. Benjamini Y, Hochberg Y. Controlling the false discovery rate: a practical and powerful approach to multiple testing. J R Stat Soc Ser B Methodol. 1995;57(1):289-300.

76. Liu Y. SHAP for XGBoost in R: SHAPforxgboosthttps://github.com/liuyanguu/ SHAPforxgboost; 2019.

\section{Publisher's Note}

Springer Nature remains neutral with regard to jurisdictional claims in published maps and institutional affiliations.

Ready to submit your research? Choose BMC and benefit from:

- fast, convenient online submission

- thorough peer review by experienced researchers in your field

- rapid publication on acceptance

- support for research data, including large and complex data types

- gold Open Access which fosters wider collaboration and increased citations

- maximum visibility for your research: over $100 \mathrm{M}$ website views per year

At BMC, research is always in progress.

Learn more biomedcentral.com/submissions 